\title{
Consideraciones clínicas y terapéuticas de las enfermedades benignas de la mama
}

\author{
Ramiro Sánchez Ramírez*
}

\begin{abstract}
RESUMEN: Durante mucho tiempo se ha debatido el nombre que se le debe dar a una serie de condiciones que puede presentar la mama femenina en el transcurso de su vida, y el término enfermedad fibroquística fue aplicado a todas estas manifestaciones caracterizadas por dolor, nodularidad, masa o la combinación de ellas. Posteriormente se empezó a utilizar el término ANDI para significar que en realidad no se trataba de una enfermedad, sino los cambios involutivos de la mama.
\end{abstract}

La gran confusión nace de tratar de correlacionar una sintomatología terapéutica específica con una patología también específica. En este artículo tratamos de diferenciar tres grupos de las enfermedades benignas de la mama, que son:

a) El grupo que responde exclusivamente a una clasificación histológica

b) El grupo que responde exclusivamente a una clasificación clínica

c) El grupo que responde a un cuadro clínico específico y a una patología consistente con el mismo.

PALABRAS CLAVES: ANDI - Enfermedad benigna de la mama, Hiperplasia epitelial atípica, Mastalgia cíclica y no cíclica, Ectasia ductal, mastitis periductal, absceso periareolar, fístula recidivante.

SUMMARY: For a long time it has been debated which name has to be given to servies of conditions has the femenine breast can present during the course of her life, and the term fibrocystic disease was applied to all those symptoms characterized by pain, nodularity, mass or a combination of these. Afterwards the term ANDI was used to mean that it wasn't really adisease but involutive changes of the breast, the great contusion is born from trying to correlate a specified therapeutic simptomatology with a specified pathology. In this article we trie to differentiate three groups in the benign diseases of the breast. They are: a) The group that responds exclusively to an histological clasification. b) The group that responds exclusively to a clinical clasification. c) The group that responds to a specific clinical feature and a consistent pathology.

KEY WORDS: ANDI - Aberrations - of mormal development and involution benign disease of the breast.

Durante mucho tiempo se ha debatido el nombre que se le debe dar a una serie de condiciones que puede presentar la mama femenina, en el transcurso de su vida, y caracterizadas básicamente por dolor, nodularidad, masa o la combinación de ellas.

Durante muchos años el término de enfermedad fibroquística fue aplicado a esta serie de patologías y en ella cabían todas las manifestaciones de la mama diferentes a cáncer, incluso para el clínico el término de enfermedad fibroquística se convirtió en la respuesta más sencilla que podía darle a su paciente. Durante muchos años se utilizó indistintamente para caracterizar patologías tan disímiles como una papilomatosis florida, un dolor premestrual o una descarga por el pezón. En octubre del 82, Love y cols (1) cuestionan el nombre de enfermedad fibroquística y muestran una revisión de la

Miembro del servicio de Cirugía de tumores de Mama, tejidos blandos y melanoma del Instituto Nacional de Cancerología.Especialista Consultor de la Liga contra el Cáncer, Seccional Bogotá. literatura (2-4) donde en estudios histológicos en biopsias de mama revisados por diferentes patólogos la mayoría de los especímenes eran clasificados como enfermedad fibroquística, demostrando que lo más probable es que se tratase más de una condición normal de la mama en diferentes estados de involución y no una enfermedad como tal.

Posteriormente en la primera y segunda conferencia nacional de enfermedades benignas de la mama en Southampton, se adoptó el término de ANDI iniciales de Aberrations of Normal Development and Involution, término que ha ganado mucha aceptación sobre todo en Europa y en el Reino Unido y está basado en el hecho de que muchos de los desórdenes dẹ la mama son aberraciones o alteraciones menores del proceso normal del desarrollo, de la respuesta hormonal cíclica y la involución que normalmente sufre la mama de la mujer durante toda su vida. En el término ANDI se incluyen los procesos benignos más importantes, como son: ectasia ductal / mastitis periductal, dolor y nodularidad y las hiperplasias 
epiteliales. El concepto también puede extenderse para cubrir un amplio espectro de desórdenes benignos que tienen diferente grado de severidad tanto histológico como de sintomatología.

El mayor problema que enfrentamos al estudiar la patología benigna de la mama agrupado bajo el término condición fibroquística, enfermedad fibroquística o ANDI, es que existen una amplia gama de presentaciones clínicas generalmente relacionadas con dolor mamario y masa; o dolor y nodularidad, o mastalgia en diferentes grados de severidad, que muestra muy poca correlación con una alteración histológica específica para cada uno de esos cuadros, por lo tanto no es posible determinar entidades clinicopatológicas precisas, y la división en categorías es difícil e improcedente (5). La nodularidad es un ejemplo típico, se encuentra en muchos exámenes físicos, y puede estar asociada tanto a condiciones benignas como a malignas, y presentarse asociada a dolor o sin el, e histológicamente puede corresponder a una adenosis esclerosante, a múltiples macroquistes con reacción fibrosa o una infiltración grasa. Por lo tanto consideramos que se hace necesario subdividir en tres grandes grupos todas estas entidades, el primero analizado desde el punto de vista de patología y que tiene interés básicamente para tratar de correlacionar estos hallazgos con la clínica pero sobre todo con el posible desarrollo de una neoplasia maligna. El segundo grupo clasificado básicamente por los hallazgos clínicos, para tratar de dar un enfoque nacional y un tratamiento terapéutico efectivo o por lo menos consistente. Podría existir un tercer grupo en los cuales de manera sistemática se encuentran relacionadas unas manifestaciones clínicas más o menos definidas y unos hallazgos histológicos claros asociados a dicha patología, como es el caso de la ectasia ductal, la mastitis de células plasmáticas, la mastitis periductal, los abscesos periareolares no asociados a la lactancia y las fístulas recidivantes o persistentes, los fibroadenomas, los macroquistes y el papiloma intraductal sintomático.

\section{Grupo I - Lesiones exclusivamente histopatológicas}

En el primer grupo, se estudiarían las lesiones desde el punto de vista exclusivamente histopatológico, y cuya denominación no se asocia con ninguna clínica específica. Generalmente son términos referidos por el patólogo en un espécimen de biopsia, llevada a cabo por la presencia de una masa o de una zona clínica o mamográficamente sospechosa de cáncer. Estas entidades a pesar de que tienen un cuadro histológico definido, no se correlacionan con un cuadro clínico preciso y el solo ejercicio histopatológico es vano si no se cuenta con una estimación predictiva desde el punto de vista clínico. En esta categoría entrarían la adenosis esclerosante, la metaplasia apocrina, la papilomatosis florida, los microquistes con reacción fibrosa, la epiteliosis, la adenosis simple y la esclerosante; algunas de estas pueden presentar hiperplasia epitelial con atipia o sin ella.

Los microquistes con reacción fibrosa: Estos quistes nacen en la porción terminal e interlobulillar de los conductos, por la proliferación activa del epitelio y probablemente responden a las mismas causas que los macroquistes y que por razones aún no bien comprendidas tienden a permanecer de un tamaño microscópico. Después de la retención de la secreción aparece secundariamente una atrofia por compresión del epitelio. Una de las teorías para tratar de explicar el por qué permanecen de ese tamaño, es la fuga de secreciones retenidas hacia el tejido conjuntivo, lo que produce una inflamación aséptica con el consiguiente proceso granulomatoso y esclerótico, característico de este proceso, y que impediría que los quistes sigan creciendo. Por lo tanto se considera que los macroquisteś no encontraron este tipo de alteraciones y lograron desarrollarse hasta un gran tamaño.

Metaplasia apocrina: La metaplasia apocrina suele ser una diferenciación especial de las células de los conductos y de los lobulillos, sin ninguna relación con el desarrollo de un carcinoma aunque ciertos investigadores han asegurado que la célula apocrina podría ser el origen de una hiperplasia regular y atípica (6). La célula apocrina se reconoce por su aspecto prismático un citoplasma granular eosinofílico núcleos basales redondeados y un vértice de forma de cúpula.

Esta metaplasia apocrina de las células epiteliales puede verse en una mastopatía simple o proliferativa, en un fibroadenoma, o en una adenosis esclerosante, e incluso algunos consideran que el carcinoma apocrino podría provenir de una metaplasia de este tipo.

La Hiperplasia epitelial benigna: Bajo esta denominación pueden agruparse una gran cantidad de alteraciones epiteliales benignas que pueden ir desde moderadas hasta floridas y que pueden tener atipia tanto en la arquitectura como en la célula misma y podrían dividirse en dos grandes grupos como son la proliferación intraductal y la extraductal. Las alteraciones proliferativas intraductales se clasificarían como epiteliosis y las extraductales como adenosis.

La Epiteliosis o Papilomatosis: Es un componente frecuente dentro de las descripciones histopatológicas de la enfermedad fibroquística o ANDI. Es una entidad microscópica específica y con patrones histológicos bien definidos, encontrándose cuatro tipos diferentes que son: el cribiforme, el fenestrado, el sólido obstructivo, y el seudopapilar.

Hay dos tipos de células que se ven básicamente en esta patología que son el tipo de célula plana o cilíndrica secretora y el tipo de célula basal o indeterminado. La epiteliosis sin atipia tiene estructuras celulares y nucleares bien regulares o bien muestra solo un ligero pleomorfismo. Las mitosis son raras (6). Más adelante hablaremos de la papilomatosis florida y el papiloma solitario.

La Adenosis: Es la proliferación extraductal y deben distinguirse dos tipos diferentes que son la adenosis simple o adenosis ductal roma y la adenosis esclerosante, de la cual hablaremos más adelante. La adenosis simple muestra en el estudio histológico en el área de los lobulillos una hiperplasia organoide, y aunque se encuentra aumentado de tamaño en todas partes, el número total de unidades terminales no está aumentado en contraste con lo que se ve o con lo que se encuentra en la hiperplasia lobulillar. 
Adenosis esclerosante: El término adenosis esclerosante es eminentemente histopatológico y se refiere a una entidad que varía ampliamente, desde la clínica hasta los hallazgos patológicos. Incluso macroscópicamente es difícil distinguirla de un carcinoma por su gran vascularización y su friabilidad. Microscópicamente es también difícil distinguirla de un carcinoma, particularmente cuando se observa una pseudoinfiltración, que consiste en células que están alrededor de las vainas nerviosas dando la apariencia de una invasión perineural. Se observa además un aumento del colágeno, y esta acumulación de fibras provoca una distorsión y una compresión de las células neoepiteliales proliferantes. En el parénquima de la adenosis esclerosante, se ven calcificaciones pequeñas y multicéntricas, a menudo microscópicas. La confluencia de focos floridos forma un engrosamiento difuso y palpable o tumores mutinodulares, y en una biopsia por congelación, para el patólogo, es muy difícil distinguir estas formaciones de un carcinoma. Clásicamente la proliferación fibrosa o el tejido mioepitelial están dispuestos en remolino lo cual distorsiona la arquitectura normal de los lóbulos y el epitelio muestra a menudo hiperplasia (7-8).

Desde el punto de vista clínico también la adenosis esclerosante presenta varias formas. Foote y Stewart (9) consideran que existen dos formas principalmente, una la de un tumor discretamente palpable y la segunda la del foco encontrado solo incidentalmente por el patólogo. La masa palpable es más común entre los 20 y 30 años, es móvil, libre, y no se acompaña de cambios en la piel.

La segunda forma es veinte a treinta veces más común y se encuentra en un rango tan amplio como es de 20 a 50 años. En el estudio de Urba y Adair (10) encontraron que el dolor y la hipersensibilidad estaban presentes en el $45 \%$ de las pacientes que presentaban adenosis esclerosantes. Es necesario tener en cuenta que la adenosis esclerosante puede simular un carcinoma tanto clínica como radiológicamente y ante la duda se debe practicar un BACAF con localización estereotáxica o la biopsia escisional.

Papilomatosis ductal: Los papilomas son proliferaciones arborescentes del sistema ductal, son prolongaciones que tienen un núcleo fibrovascular cubiertos por un epitelio de diferenciación variable.

Los papilomas multicéntricos afectan los conductos de tamaño medio y más pequeño y raramente presentan secreciones patológicas. El gran papiloma solitario es una entidad específica localizada en la parte distal subareolar de los conductos galactóforos, y presentan un síntoma específico que es la secreción serosa o sanguinolenta por el pezón. En la periferia las lesiones papilares a menudo son múltiples y se continúan con alteraciones hiperplásicas en el interior de las unidades lobulillares, como lo han demostrado Ohuchi y col (14). En los casos en que los papilomas son muy extensos puede encontrarse asociada una hiperplasia atípica y en algunas ocasiones un ca ductal un situ (16). De hecho muchos consideran que estas pacientes tienen un riesgo mayor de desarrollar un carcinoma ulterior (17-19).

Hiperplasia atípica: Todas las anteriores descripciones son eminentemente histopatológicas, y no tendrían ningún significado si el patólogo no le agrega el término "con hiperplasia atípica", que incluye todas las proliferaciones patológicas del sistema ductal y lobulillar. De hecho para el clínico no existiría ninguna indicación de conducta terapéutica si en el espécimen de una biopsia por un nódulo sospechoso clínicamente o mamográficamente, en el reporte patológico le informan adenosis esclerosante. La situación variaría enormemente si además de lo anterior se coloca "Hiperplasia Atípica".

Este término de "Hiperplasia Atípica", tiene por finalidad indicar un grupo de patrones histológicos relativamente específicos que no, solamente son atípicos, sino que además se asocian con un riesgo aumentado de desarrollo de cáncer mamario en una fase ulterior (15).

Las lesiones hiperplásicas atípicas presentan algunos de los rasgos de los carcinomas in situ, pero carecen de un elemento mayor que defina el verdadero carcinoma. Es muy difícil evaluar el verdadero poder premaligno de las lesiones benignas del seno, pero el estudio de Nashville (20) mostró que de 10.000 biopsias evaluadas $70 \%$ tenían como diagnóstico histológico tejido mamario benigno. El 30\% restante revelaban enfermedad proliferativa. Estas lesiones se caracterizaban por una hiperplasia de grado moderado por lo menos y que se sociaban con un riesgo por lo menos dos veces mayor. En esta categoría estaban incluidas la hiperplasia ductal habitual de grados moderado y florido, así como la adenosis esclerosante y los papilomas. Posteriormente se creó la categoría de la hiperplasia atípica para caracterizar las lesiones que cumplían algunos criterios de carcinoma in situ pero no todos. Se considera que son entre el 4 y $7 \%$ de todas las hiperplasias y se asocia con un riesgo de cáncer de mama entre 4 a 5 veces mayor.

Existen dos subtipos morfológicamente distintos de hiperplasia atípica, conocidos como hiperplasia lobulillar atípica a hiperplasia ductal atípica. El riesgo de cáncer de mama es similar en ambas lesiones.

En la actualidad a estas pacientes las seguimos muy de cerca tanto mamográfica como clínicamente la resección. Un grupo especial son las pacientes con alto riesgo de desarrollar carcinoma e historia familiar del mismo y en las cuales se presenta una hiperplasia atípica extensa, llegándose a considerar un tratamiento más agresivo en este grupo.

\section{Grupo dos - cuadro eminentemente clínico}

Este grupo trata de ver la patología mamaria de acuerdo a la sintomatología clínica que presenta, tratando de darle más un enfoque fisiológico metabólico y hormonal. Se trata de explicar toda la patología a través de mecanismos de disbalance hormonal.

La doctora Susan Love (11) ha definido de la siguiente manera: A) la mastalgia cíclica B) la mastalgia no cíclica C) nodularidad fisiológica y D) el dolor referido a partir de la unión condrocostal o del cuello.

Mastalgia cíclica: Se cree que las hormonas desempeñan un papel importante en la presentación de esta sintomatología y se ha implicado un disbalance hormonal en la producción de la misma. Se dice que los ovarios no producen exactamente la misma cantidad de estrógeno todos los meses, y es factible que el tejido mamario sea sensible a estas alteraciones. En términos generales se 
considera que estas pacientes sólo les basta un examen clínico concienzudo, una mamografía si es mayor y una orientación médica adecuada, explicándoles que no padecen ninguna enfermedad grave; probablemente un $80 \%$ de las pacientes mejorarán descartándoles la presencia de cáncer y ocasionalmente formulándoles un analgésico suave. Un pequeño grupo de pacientes puede presentar persistencia de su dolor y puede requerir el uso de fármacos que alteran el medio hormonal. Como es el caso del Danazol, derivado sintético de la 17 etiniltestosterona cuya eficacia ha sido confirmada en varios estudios. Usando dosis de 200 a $400 \mathrm{mlgs}$. día, se obtiene una respuesta del $70 \%$ en pacientes con mastalgia cíclica, pero la recaída es frecuente al abandonar la droga y un $20 \%$ presentan efectos colaterales, como son trastornos menstruales, aumento de peso, aumento del vello facial y cambios en la voz. (12).

También se ha utilizado con éxito el Nafarelin un análogo de la LHRH, cuya continua administración causa una baja en la producción pituitaria de LH y FSH, y por lo tanto causa cesación de la producción hormonal sexual ovárica, produciendo un efecto dramático sobre la sintomatología (13).

Otros fármacos utilizados en el manejo de la mastalgia cíclica son la bromocriptina, que es un antagonista de la prolactina, y que aunque está demostrado que disminuye el dolor a la palpación y la nodularidad, su uso debe ser prolongado y por lo tanto sus efectos secundarios como son náuseas, cefaleas y mareos se hacen prácticamente más intolerables que la misma mastalgia. También se ha utilizado el tamoxifén y la progesterona con resultados favorables especialmente en el primero.

Mastalgia no cíclica: Esta sintomatología se caracteriza por presentar un dolor no relacionado con el ciclo menstrual; generalmente es un sitio o máximo dos donde el dolor es constante, responde este dolor parcialmente a los analgésicos pero una vez pasa el efecto de los mismos vuelve a presentarse; el tratamiento hormonal no surte efecto. Aunque no hay una explicación exacta para el mismo se considera que la fibrosis puede haber involucrado terminaciones nerviosas que explicarían el dolor persistentemente localizado. Es necesario descartar en estas pacientes un cáncer y si es mayor se debe realizar una mamografía.

Normalmente con unas palabras tranquilizadoras y un analgésico las pacientes mejoran durante un largo período. Algunos autores aconsejan la infiltración de un analgésico local; terapéutica en la cual no tenemos amplia experiencia y que en algunas ocasiones se ha asociado a mastitis inespecífica.

Nodularidad fisiológica: Un porcentaje no muy grande de pacientes puede presentarse con múltiples protuberancias en los senos que pueden ir desde nodulaciones muy finas como pequeños granos de arena hasta nódulos de mayor tamaño incluso de 1 o $2 \mathrm{cms}$. La nodularidad en sí no da dolor pero en algunas pacientes se puede presentar y debe ser clasificado como dolor cíclico o no cíclico. El mayor problema de estas pacientes es descartar la presencia de un cáncer ya que tanto clínica como mamográficamente puede pasar desapercibido. Estas mamas tienen tendencia a tener gran cantidad de fibrosis lo que complica aún más su diagnóstico. En este grupo de pacientes el autoexamen de mama y la mamografía de alta resolución pueden llegar a ser métodos importantes en el diagnóstico temprano del C:A.

Dolor referido: El dolor referido más común asociado a mastalgia es el de la condritis costal (síndrome de Tietze); es también un dolor no cíclico y se debe a la inflamación de la unión costocondral y que puede ocurrir de modo expontáneo o después de un trauma o un movimiento brusco que generalmente pasa desapercibido. A la palpación profunda del cartilago condrocostal hay un dolor exquisito. También la radiculopatía cervical cuya principal característica clínica es extenderse hacia el cuello y a lo largo del borde externo del brazo. Otros dolores referidos pueden estar asociados a infartos, anginas, hernia hiatal, colelitiasis etc.

En términos generales, la mayor parte de las pacientes que consultan por mastalgia, mejoran dramáticamente de su sintomatología con la toma de la mamografía y al escuchar la explicación del médico de que no tiene cáncer. Solo un pequeño porcentaje puede requerir un tratamiento más enérgico, pero siempre debemos tener en mente que lo principal es descartar una neoplasia.

\section{Grupo tres-lesiones con correlación clínica e histológica}

En este grupo revisaremos las lesiones que de manera consistente presentan un cuadro clínico específico y un cuadro histológico asociado al mismo. En esta categoría incluimos al fibroadenoma, a los macroquistes, a la ectasia ductal, la mastitis de cálulas plasmáticas, la mastitis periductal, la mastitis química, los abscesos subareolares, las fístulas recidivantes crónicas y el papiloma intraductal. Este último fue revisado en las papilomatosis porque con cierta frecuencia es un hallazgo histológico, pero también es verdad que presenta una sintomatología específica como es la secreción sanguinolenta por el pezón, además se puede diagnosticar a través de una citología de la secreción. Nos dedicaremos básicamente a revisar la ectasia ductal y sus complicaciones y los abscesos subareolares y las fístulas recidivantes.

Fibroadenomas: Los fibroadenomas a menudo se presentan como una masa palpable, móvil bien definida y rara vez fija al tejido adyacente. El aspecto macroscópico es por lo general típico y casi siempre diagnóstico, observándose una clara delimitación del tejido mamario circundante, de color blanquecino o marrón claro, con una superficie, con una fibrosis muy densa del estroma y ocasionalmente calcificaciones. Microscópicamente también presenta un cuadro muy característico caracterizado por tejido fibroso que rodea estructuras epiteliales ductales redondeadas y fácilmente identificables (fibroadenoma de tipo pericanalicular) o el epitelio puede estar estirado en estructura de configuración curvilínea (fibroadenoma de tipo intracanalicular).

Los fibroadenomas crecen 2 ó 3 centímetros y generalmente cesan su crecimiento. El término fibroadenoma se refiere a un tumor benigno y delimitado de la mama que presenta una combinación de elementos glandulares y mesenquimáticos. Existen una serie de variantes de 
esta definición y que por lo tanto se reconocen como entidades distintas como son el amartoma, el adenoma de la lactancia, el adenolipoma, el adenoma tubular, el adenoma gigante y el fibroadenoma juvenil. La caracterización de cada uno de ellos se nos sale de las posibilidades de este artículo. El desarrollo ulterior de cáncer a partir de un fibroadenoma típico no ha sido considerado mayor que el de la población general.

Macroquistes: Los macroquistes ocurren en el $7 \%$ de las mujeres adultas. En hallazgos de autopsia, se ha encontrado que hasta el $21 \%$ tienen quistes en las mamas de más de 1 c. (21). La explicación de la formación de quistes es dada básicamente por el aumento de la edad de la paciente, se incrementa también el número de dilataciones acinares quísticas, las cuales pueden involucionar completamente o pueden coalescer formando grandes quistes. Esto también fue demostrado por Azzopardi (22). Se han hecho estudios del contenido líquido de los quistes subdividiéndolos de acuerdo a este resultado. Clínicamente tienen un cuadro bien definido: se presen$\tan$ mujeres entre 40 y 55 años de edad en un $75 \%$. Solo el 7 al $10 \%$ de las mujeres desarrollan un quiste antes de los 35 años. Usualmente la paciente refiere la aparición de una masa relativamente reciente, acompañada de dolor en un $30 \%$ de los casos y cerca de un $2 \%$ de los mismos tienen secreción por el pezón y en algunas ocasiones puede demostrarse comunicación con el quiste. Normalmente practicamos una aspiración de los mismos y rutinariamente los mandamos a estudio citológico aunque la rentabilidad diagnóstica es muy baja. Si el macroquiste se reproduce en un período relativamente corto preferimos realizar una extirpación del mismo o realizamos una neumocistografía.

\section{Ectasia ductal}

El término de ectasia ductal está asociado con la dilatación de los conductos. Sin embargo, la mayoría de los actores actuales al término para designar a una entidad o una serie de entidades las cuales se asocian a manifestaciones clínicas como dolor, cicatrización, retracción del pezón e incluso masa palpable en la región mamaria retroareolar. La dilatación de los conductos como tal es practicamente asintomática y pasa desapercibida, y es una condición sumamente frecuente. Cuando se asocia a manifestaciones clínicas como las anteriormente descritas se torna sintomática y es a este tipo de lesiones al que se aplica el nombre de ectasia ductal.

Existen múltiples nombres para referirse a esta condición como son mastitis periductal, mastitis de células plasmáticas, comedomastitis, mastitis química, mastitis granulomatosa, mastitis obliterante, etc. (23).

Los conductos generalmente están comprometidos segmentariamente, afectando a los conductos adyacentes que se extienden hacia el interior de la mama en forma divergente (16).

Etiología y patogénesis: Algunos de los factores implicados en la mastitis periductal incluyen embarazos y lactancia, dilatación ductal, e invasión bacteriana. Ultimamente también se ha sugerido que este proceso puede tener un fenómeno autoinmune como base (24). Se han propuesto básicamente dos teorías, la primera que postula que la enfermedad comienza con la dilatación de los conductos colectores, que son distendidos por restos celulares y material que contiene lípidos. En esta fase inicial no hay sintomatología y la enfermedad no es detectada. Si la enfermedad progresa comienza a aparecer secreción parduzca o amarillenta por el pezón, las paredes de los conductos sufren un gran engrosamiento por la fibrosis y la infiltración inflamatoria. Esta fibrosis no solamente engruesa las paredes, sino que también las acorta, lo que provoca un aplanamiento y finalmente la retracción del pezón. El epitelio atrófico del conducto se rompe en algunos lugares y el material lipídico irritante provoca una reacción inflamatoria similar a la de la esteatonecrosis, y en algunos casos hay predominio de células plasmáticas. Esto es un cuadro de una fase tardía de la ectasia del conducto mamario (6).

Recientemente, este punto de vista ha sido puesto en duda (25-27), se ha sugerido que la inflamación periductal es el rasgo esencial y primario, resultando una dilatación del conducto a partir de la destrucción de la lámina elástica que soporta al mismo. Esta teoría está soportada en hallazgos de patología, en biopsias de pacientes de diferente edad, que mostraron mastitis periductal en pacientes jóvenes y mayor cantidad de ectasia ductal en pacientes mayores, lo que sugiere que la mastitis periductal y la ectasia ductal son componentes de una misma enfermedad, siendo la mastitis periductal el evento inicial y la ectasia el resultado.

Rasgos clínicos y presentación: En las fases iniciales de la enfermedad, no hay ningún síntoma en absoluto. Cuando han aparecido las fibrosis y las alteraciones inflamatorias, puede haber una secreción espontánea por el pezón y más tarde una retracción de éste. Finalmente se desarrolla una base irregular detrás de la areola, que simula un carcinoma. En un momento u otro durante la evolución de la enfermedad, la paciente con ectasia del conducto, tiene a menudo signos clínicos de inflamación, tales como dolor espontáneo o a la presión sobre el tumor, ligero enrojecimiento o edema de la piel suprayacente, a menudo intermitentes, o una elevación de la temperatura del cuerpo.

Algunas veces, en las fases inflamatorias finales de la ectasia del conducto, puede formarse un absceso acompañado de todos los signos habituales: enrojecimiento y calor locales e incluso edema de la piel. Sin embargo, estos abscesos que evolucionan a partir de una ectasia del conducto suelen ser menos dolorosos y de más bajo grado que los abscesos corrientes asociados a la lactancia.

La edad de presentación es variable puede iniciarse desde los treinta años con un pico de incidencia entre los cuarenta y cincuenta años, los signos y síntomas varían según el grupo de edad y el estado de la patología subyacente.

El dolor se encuentra más en pacientes jóvenes y está asociado a la mastitis periductal y a la inflamación que produce. Este dolor puede preceder a la aparición de una masa de características inflamatorias o puede ser un síntoma aislado, la frecuencia en la cual la mastitis perductal causa dolor en el seno y es desconocida, algunos investigadores piensan que esta inflamación puede 
estar relacionada con una actividad bacteriana específica y que los antibióticos pueden ayudar.

También la mastitis puede presentarse como una masa y como causa de masa palpable es diferente la frecuencia de acuerdo a los grupos de edades. Se ha calculado que el 3 al $4 \%$ de todas las masas benignas del seno en términos generales, aunque es el responsable del $12 \%$ de las masas benignas del seno en mujeres por encima de los 55 años de edad. Las masas se presentan usualmente en los márgenes periareolares, y frecuentemente está asociado con eritema de la piel abyacente sobre todo en mujeres jóvenes. La patología de estas pacientes muestra una intensa mastitis periductal. Los ductos en sí mismos no están dilatados pero alrededor de ellos hay polimorfos y/o células plasmáticas, linfocitos, células gigantes y no es infrecuente granulomas. En las pacientes mayores con masa, la histología es bien diferente, los ductos están dilatados, hay fibrosis y una pequeña inflamación periductal.

15 a $20 \%$ de las pacientes presentan descarga por el pezón asociado a mastitis periductal. El color de la descarga varía lo mismo que su consistencia y puede ser cremosa, amarillenta, verdosa o café rara vez tiene aspecto sanguinolenta, aunque más de la mitad de estas descargas son positivas para sangre en un test de prueba (28-29).

En pacientes jóvenes la descarga tiende a ser delgada y a menudo acuosa mientras en las pacientes mayores tiende a ser más viscosa y pastosa. La descarga puede ser bilateral o unilateral y puede ser a través de uno o de varios ductos, y puede ser además multicoloreada.

La retacción del pezón varía de acuerdo a la evolución de la enfermedad y puede ser muy leve en los estadios tempranos de la enfermedad (30) y está presente en el $75 \%$ de las pacientes que sufren una inflamación periareolar (31). La retracción marcada o la inversión del pezón ocurre más tardíamente y se debe a la retracción de los ductos, la retracción es bilateral en el $15 \%$ de los casos (30).

Diagnóstico y tratamiento: Desde el punto de vista radiológico, la mamografía tiene unas imágenes bien reconocidas de esta patología. La ectasia ductal se puede presentar en el $11 \%$ de las mujeres a las que se le hace una autopsia. El problema clínico más complejo en la práctica es diferenciar una ectasia ductal de un carcinoma, al cual puede simular. Un aumento de los ductos, calcificaciones asociadas, cambios inflamatorios, retracción del pezón y masas radiológicas pueden ocurrir con o sin acompañarse de cambios de hipervascularidad o de engrosamiento de la piel. Todos estos rasgos radiológicos pueden estar asociados a una masa palpable clínicamente o a no encontrar nada en el examen físico. La ectasia ductal se ve mamográficamente como unas bandas densas (algunas mayores de $3 \mathrm{~mm}$ ) que convergen hacia el pezón y el cual puede estar o no retraido. Menos frecuentemente se ve como una banda traslúcida debido al alto contenido de grasa de las secreciones. Casi siempre la ectasia ductal en la mamografía es bilateral, así clínicamente no se detecte si no en uno de los lados. Cuando radiológicamente hay dilatación de un solo lado el radiólogo debe estar acucioso y ser cuidadoso en su diagnóstico ya que hay que excluir un carcinoma intraductal o una papilomatosis en tales casos (33).

Las calcificaciones son frecuentes y sus características son bien definidas en esta patología, con frecuencia son gruesas y de contornos lisos y pueden ir de unas pocas a muy numerosas. Tienden a seguir las ramificaciones de los conductos. Los cambios inflamatorios de la mastitis periductal son más frecuentemente encontrados en la región retroareolar donde convergen los ductos, se observa un aumento de la densidad a este nivel, ocasionalmente si se toma la radiografía en una fase aguda del proceso puede verse un engrosamiento de la piel debida al edema y en algunos casos una imagen de llamarada por los ductos edematizados que se internan en el tejido mamario adyacente.

El BACAF, que se hace a las masas asociadas en la mastitis periductal presenta algunos rasgos histológicos característicos que permiten un diagnóstico definitivo, como son la presencia de abundantes polimorfos nucleares, células plasmáticas, linfocitos y células gigantes en las masas de las pacientes jóvenes con mastitis periductal (24). En las pacientes mayores el diagnóstico citológico es más difícil ya que con frecuencia es acelular, conteniendo solo detritus y ocasionalmente células gigantes que no sirven como tal para hacer un diagnóstico específico.

Cuando existen masas pobremente definidas o bien definidas es mandatorio el BACAF, y si existe sospecha de cáncer clínica o radiológicamente, debe hacerse una biopsia de la zona sospechosa.

El tratamiento de la fase aguda de las masas periareolares asociadas a esta patología es poco satisfactorio. Los antibióticos de amplio espectro no han dado buenos resultados (15), y la intervención quirúrgica puede ser complicada por la infección de la herida. Un reciente estudio sugiere que el metronidazol combinado con cefradina es efectiva resolver esta inflamación. En términos generales debe evitarse la biopsia en pacientes jóvenes y tratar de manejarlas con estas combinaciones de antibióticos. Ocasionalmente en las pacientes mayores se presentan abscesos a repetición, que terminan deformando el complejo areola pezón y se torna una condición sumamente dolorosa, por lo que una vez se resuelve la infección preferimos practicable una extirpación de los conductos terminales evertiendo el pezón, utilizando antibióticos del tipo metronidazol más tetraciclina en combinación, por un largo período tanto pre como pos operatorio, tratamiento que nos ha resultado efectivo en nuestro hospital.

\section{Absceso subareolar recidivante y fístula}

El absceso subareolar recidivante es una enfermedad relativamente frecuente y de difícil manejo en la mama de la mujer joven no tiene relación con el embarazo ni con la lactancia. La historia de este proceso comienza con un absceso pariareolar que ha sido tratado con múltiples incisiones al parénquima mamario mejorando durante algún tiempo y volviendo a aparecer luego. Otros nombres con que se conoce este proceso son: absceso subareolar crónico, fístula de un conducto galactóforo o fístula de un conducto mamario. 
La enfermedad no tiene ninguna relación con el embarazo ni con la lactancia y a menudo comienza como una zona de inflamación subareolar que evoluciona hacia un pequeño absceso en el borde de la areola, si se abre y drena o si espontáneamente se drena, el proceso parece desaparecer para que unos meses más tarde se reactive. Posteriormente puede presentarse una fístula que drena de una manera espontánea e intermitentemente. A esta altura, se ha establecido una comunicación entre el absceso que no es más que un conducto galactóforo dilatado e infectado en la piel. Algunas veces hay drenaje simultáneo de pus tanto por el orificio pustuloso como por el conducto galactóforo afectado a nivel del pezón. En un número importante de casos coexiste un pezón invertido.

Para poder comprender exactamente la patología de este trastorno, es importante estar familiarizado con la anatomía del pezón y de los conductos galactóforos. Los conductos galactóforos mayores normalmente están revestidos por un epitelio cilíndrico bajo o cuboide, en doble capa. Debajo de la superficie del pezón, cada conducto se dilata para formar una ampolla terminal y luego se estrecha ligeramente para abrirse sobre la superficie del pezón. Al principio de la ampolla, el epitelio cilíndrico es bruscamente sustituido por una capa escamosa estratificada que reviste el resto de la ampolla hasta el orificio del conducto. En los abscesos mamario subareolar recidivante, el examen histológico revela un conducto enormemente dilatado, situado dentro o inmediatamente detrás del pezón, revestido de epitelio escamoso estratificado y taponado por restos de queratina. En algún punto hay una ruptura de la pared de este conducto que comunica con el absceso subareolar. En esta enfermedad el epitelio escamoso, que normalmente se encuentra en la porción terminal y más ancha de los conductos dentro del pezón, reviste al conducto afectado en una considerable distancia dentro de la región subareolar. Se ha pretendido que esta metaplasia escamosa anormal del revestimiento del conducto que conduce al área del absceso es la clave de la historia natural de esta patología. La incisión y drenaje, o la escisión simple y local del absceso y la fibrosis que lo circunda, no elimina la fuente de infección y la recidiva es la regla, mientras no se extirpa completamente todo el trayecto fistuloso'y la parte distal del conducto.

El absceso se trata canalizando la fístula con un conducto lacrimal exteriolizándolo a través del pezón, luego se incide y extirpa completamente el tejido comprometido sobre la sonda incluyendo la parte distal del conducto. La cicatrización se produce normalmente a las tres o seis semanas a menudo con buenos resultados cosméticos. Si existe algún tipo de deformidad después de la cirugía, se deben esperar por lo menos seis meses antes de intentar hacer cualquier tipo de reconstrucción por menor que sea. Cuando una paciente viene por primera vez con un absceso subareolar, se recomienda tratarlo con una incisión y drenaje. Cuando se han producido dos o tres recidivas y se ha reconocido el verdadero carácter fistuloso de la enfermedad, es aconsejable un tratamiento más radical. Las bacterias que más se han asociado a esta patología son los estreptococos y el estafilococo áureo. El tratamiento específico para estos debe ser instaurado.

\section{Conclusión}

Es difícil tratar de evaluar la patología mamaria aún en la actualidad y con los conocimientos que poseemos. Sabemos que la mama es un órgano blanco hormonal y que sufre muchas variaciones de este tipo en el transcurso de la vida. Sin embargo, debemos racionalizar la terapéutica de nuestras pacientes, ya que es difícil decirle a una paciente que consulta por un dolor intenso en su mama "ud tiene una involución de su mama y no una enfermedad", el dolor es real, la sintomatología es real y solo espera una ayuda de su médico.

\section{BIBLIOGRAFIA}

1. S. Love, RS. Gelman, W. Silen: Fibrocystic disease of the brest- a nondisease? the N.E.J. of Medicine 1982; 16: 1010-1014.

2. Davis HH., Simons M., Davis JB. Cystic disease of the brest; relationship to carcinoma. Cancer 1964; 17: 957-978.

3. Sloss PT., Bennett WA., Clagett OT. Incidencia in normal brests of features associated with chronic cystic mastitis. Am J. Pathol 1957; 33; 1181-1191.

4. Foote FW., Stewart FW. Comparative studies of cancerous versus noncancerous breast. Ann Surg: 1945; 121: 6-53, 197-222.

5. Page DL., Simpson JF. The brest: WB. Saunders Co. Philadelfia 1991.

6. Strombeck JO., Rosato FE. Cirugía de la Mama: Salvat Editores S.A. Barcelona 1989.

7. Dawson EK. Fibrosing adenosis: A litte recognized mammary picture. Edin. Med. J. 1954; 61: 391.

8. Davies JD. Neural invasion in benign mammary dysplasia: J. Pathol; 1973; 109: 225 .
9. Foote FW., Stewart FW. Comparatives studies of cancerous versus non-cancerous breasts: Ann Surg. 1945; 121: 6.

10. Urban JA., Adain FE. Sclerosing adenosis: Cancer 1949; 2: 625.

11. Love SM. Enfermedades mamarias benignas: Patient Care 21:30 Mayo 91.

12. Maddox PR., Harrinson BJ., Mansel RE. Low-dose Danazol for mastalgia. The B: J: of Cl. Pract: Suplm. 1991; 68: 45-47.

13. Roberts JV. Experience in the use of Nafarelin for treatmen of benign breast disease. The B. J. Of Clin. pract. suplem. 1991; 68: 37-42.

14. Ohuchi N., Abe R., Takabachi T. y Tezukaf. Origin and extension of intraductal papillonas of the breast. A trhee dimensional reconstruccion satudy: Brest Cancer Reserh. Treat: 1984; 4: 117-128.

15. Page DL., Dupont WD., Rogers LW. Atypical Hyperplastic lesions of the female brest, A long term Follow-up study. Cancer 1985; 55: 2698-2708. 
16. Bland IK., Copeland III EM. The Brest; Comprehensive management of benign and malignant disease. W.B. Saunders Co. Philadelphia 1991.

17. Haagensen CD., Lane N., Lattes R., Bodian C. Lobular neoplasia (Socalled carcinoma in situ) of the brest. Cancer 1978; 42: 737-769.

18. Ohuchi N., Rikiya A., Kasai M. Possible cancerous change of intraductal of papilomas of the brest: a 3D reconstruccion study of 25 cases. Cancer 1984; 54: 605-611.

19. Salhany KE., Dupont WD., Rogers LW., Page DL. Epithelial proliferative lesions in bening intraductal papillomas. Abstract Lab Invest 1988; 58: 80A

20. Dupont WD., Page DL. Risk factors for brest cancer in women with proliferative brest disease. N. Engl. J. Med. 312: 146-151.

21. Hughes LE., Bunded NS. Brest Macrocysts WJ. Surg. 1989; 13: 711 714.

22. Azzopardi JG. Problems in brest pathology. WB. Saunders 1979.

23. Barth V., Prechtel K. Atlas de patología de la glándula mamaria. Ed Md Panamericana. 1991.

24. Dixon JM. Periductal mastitis a etiology and management. The B. J. of Cli. Pract.-Suplem. 68.

25. Azzopardi JG. Problems in Breast Pathology, London, WB. Saunders, 1979; 72-87.

26. Dixon JM., Anderson TJ., Lumsden AB., Elton RA., Roberts MM., Forrest APM. Mammary duct ectasia. Br. J. Surg. 1983; 70: 601.
27. Bonser GM., Dosset JA., Jull JW. Human and Experimental Breast Cancer, London, Pitman Medical, 1961; 338-345.

28. Geschickter CF. Diseases of the Breast, Philadelphia, Lippincott, 1948.

29. Haagensen $C D$. Mammary duct ectasia: A disease that may simulate carcinoma. Cancer 1951; 4: 749.

30. Rees BI., Gravelle IH., Hughes LE. Nipple retraction in duct ectasia. Br. J. Surg. 1977; 64: 577.

31. Dixon JM., Lee ECG., Greenall MJ. Treatment of periareolar inflammation associated with periductal mastitis using metronidazole and flucloxacillin: A preliminary report. Br. J. Clin. Practice 1988; 42: 78.

32. Sandison AT. A postmortem study of the adult breast. MD thesis, University of St. Andrews 1957.

33. H Gravelle. The radiology of periductal mastitis, The Bri. J. Clin. Practice- Supplement 68.

34. Zusca JJ., Crile F., Ayres WW. Fistulas of lactiferous ducts. Am. J. Surg. 1951; 81: 312.

35. Davies JD. Histological study of mammae in oestrogenized rats after mammary isoimmunization. Br. J. Exp. Pathol. 1972; 53: 406.

36. Hadfield J. Excision of the major duct system for benign disease of the breast. Br. J. Surg. 1960; 47: 472. 